\title{
Cardiac myocardial perfusion imaging with new SPECT cameras: Comparing apples and oranges
}

\author{
R. Glenn Wells, PhD, FCCPM, ${ }^{\mathrm{a}}$ and Terrence D. Ruddy, MD, FRCPC, FACC ${ }^{\mathrm{b}}$ \\ a Division of Cardiology, University of Ottawa Heart Institute, Ottawa, Canada \\ b Department of Cardiology, University of Ottawa Heart Institute, Ottawa, Canada
}

Received Apr 9, 2019; accepted Apr 9, 2019

doi: $10.1007 / \mathrm{s} 12350-019-01729-5$

\section{See related article, pp. $1261-1269$}

New SPECT cameras have been introduced in the last decade which are specifically designed for cardiac imaging. These cardiac SPECT cameras have improved sensitivity with maintained or improved spatial resolution $^{1}$ that has led to reduced acquisition times, lower patient doses, and enhanced image quality. Two of these new cameras are based on solid-state cadmium-zinctelluride (CZT) detectors., ${ }^{2,3}$ Although they have improved performance for myocardial perfusion imaging, they also have certain limitations, such as a very restricted field-of-view in the case of the multiple pinhole system, which preclude use for general nuclear medicine applications. In an effort to bring some of the benefits seen with the cardiac systems to more generalpurpose protocols, a conventional dual-headed gamma camera design that employs CZT detectors with matched collimation was introduced. This multi-purpose CZT-based camera may still be used for cardiac imaging and so it is of interest then to see how its performance compares to the cardiac SPECT systems. Such a performance comparison was the aim of the study presented in this issue of the journal. ${ }^{4}$ The authors present some excellent data, but care must be taken in its interpretation.

\footnotetext{
Reprint requests: R. Glenn Wells, Division of Cardiology, University of Ottawa Heart Institute, Ottawa, Canada; gwells@ottawaheart.ca J Nucl Cardiol 2020;27:1270-3.

$1071-3581 / \$ 34.00$

Copyright (c) 2019 American Society of Nuclear Cardiology.
}

\section{CZT VERSUS NAI DETECTORS}

At first glance, the difference between the multipurpose CZT-based camera and the conventional NaIbased multi-purpose camera is just the detector material itself. CZT has certain advantages. Because the detector is a solid-state device with direct conversion of the incident gamma ray into an electronic signal, it has inherently better energy resolution. A caveat to this though is that the shape of the energy response is asymmetric with a larger number of gamma rays measured as having reduced energy. Because of the low-energy tail, the photopeak energy window is still generally set to a standard $140 \mathrm{keV} \pm 10 \%$ for imaging Tc99m so as to record these unscattered photons detected with reduced energy. Therefore, the advantage of the improved energy resolution for Tc99m-based MPI is small; the scatter fraction is less but still $30 \%$ compared to $34 \%$ with a standard NaI-based camera. ${ }^{2}$ As with a standard gamma camera, a narrower energy window could be chosen which would reduce the measured scatter but also reduce the measured number of unscattered photons. A second potential advantage is that CZT is a denser material than $\mathrm{NaI}\left(5.81 \mathrm{~g} / \mathrm{cm}^{3}\right.$ vs $\left.3.67 \mathrm{~g} / \mathrm{cm}^{3}\right)$, giving it greater stopping power for $140 \mathrm{keV}$ photons. However, the intrinsic efficiency of a standard 3/8" (9.5 mm) NaI crystal is $93 \%$ whereas a $5 \mathrm{~mm}$ CZT crystal (used in the cardiac camera) stops $88 \%$ of photons and a $7.25 \mathrm{~mm} \mathrm{CZT} \mathrm{crystal} \mathrm{(used} \mathrm{in} \mathrm{the}$ general-purpose machine) stops $96 \%$. Thus, the stopping power of the detector is very similar for all three systems and it is not the crystal per se that gives the advantages seen in sensitivity. Instead, the benefits in sensitivity come primarily from the change in camera design: the cardiac camera uses 19 detector heads and pinhole collimation, while the multi-purpose CZT camera has 2 heads with matched parallel-hole collimators. 


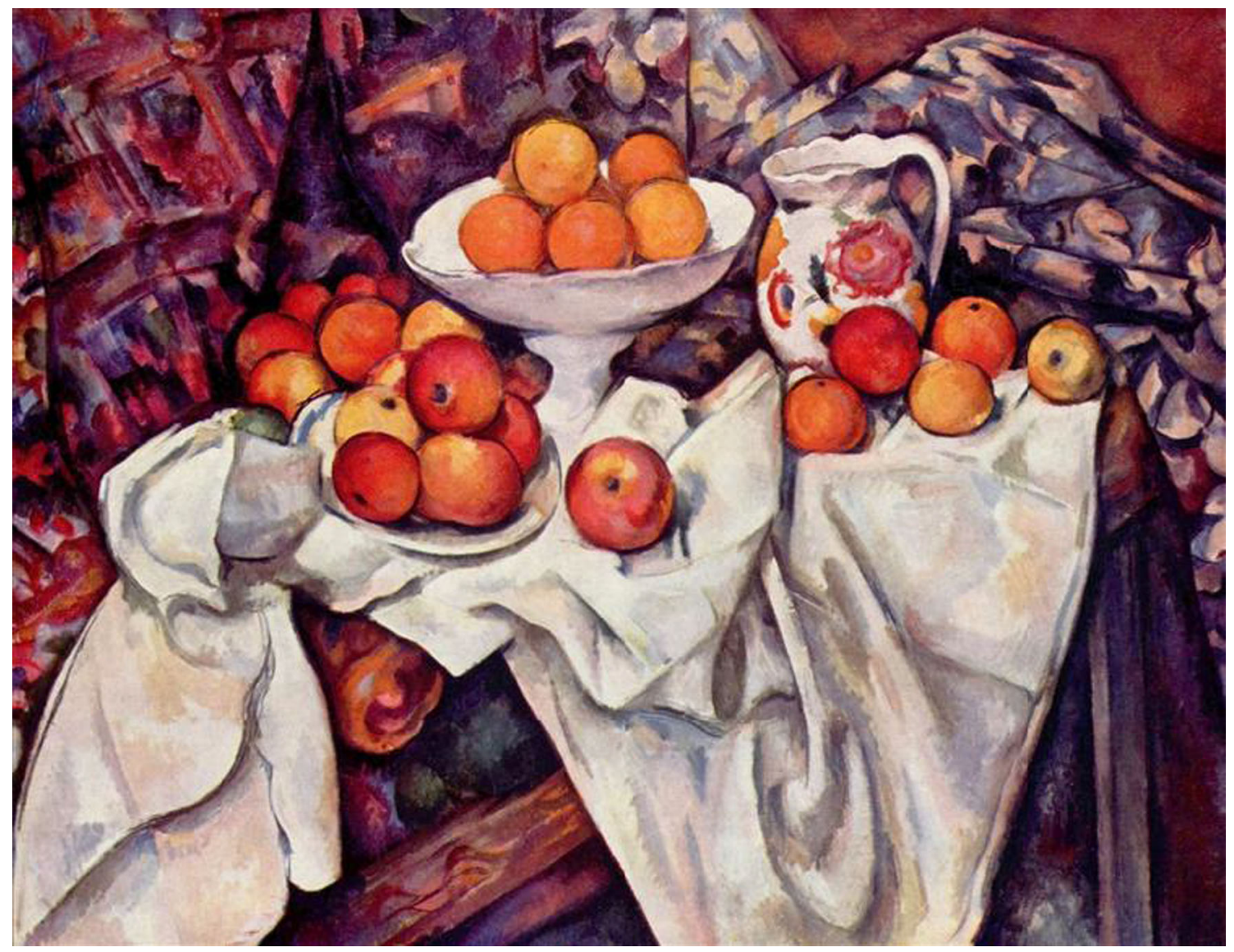

Figure 1. Paul Cézanne (1839-1906) Apples and Oranges (Circa 1899 Oil on canvas H. 74; W. 93 cm) Musée d'Orsay, Paris, France.

\section{EFFECTS ON COUNT SENSITIVITY AND SPATIAL RESOLUTION}

The difference in collimation introduces the first challenge in performing a comparison. With parallelhole systems, sensitivity is not dependent on the distance of the source to the collimator and thus is relatively constant over the FOV of the camera. With pinhole collimators, sensitivity is strongly distance-dependent, leading to highly variable system sensitivity across the FOV. ${ }^{5,6}$ Measuring the counts acquired per $\mathrm{MBq}$ of a source in the cardiac system is valid, but only for that particular size, shape and location of source. To further complicate the comparison, sensitivity in clinical MPI also depends on the attenuation of the object. For a parallel-hole system, the effects of attenuation are constant if the object is moved through the FOV, but for the pinhole system, the effects change depending on where in the FOV the object is because this changes what part of the object the photons travel through to reach the detector. ${ }^{7,8}$
Another commonly used metric of image quality is spatial resolution. NEMA recommends using filtered back projection (FBP) reconstruction for creating images to measure reconstructed spatial resolution. FBP is good for this purpose because it is a linear algorithm and so reconstruction of the point sources does not depend on background activity. However, nuclear medicine has generally moved to iterative reconstruction (IR), most commonly OSEM, instead of FBP because iterative algorithms can perform better with low-count data and allow accurate attenuation correction and collimator modelling. Indeed, FBP is not available to reconstruct images on the pinhole cardiac camera. Thus, resolution measured with IR images is much more clinically relevant than resolution of FBP images. However, IR is non-linear and so image noise and resolution depend on the number of iterations used, location within the FOV, and the distribution of source activity. Thus, while it is more clinically relevant to compare resolution with OSEM images, results will 
depend on the specifics of the reconstruction parameters, algorithm used, and the activity distribution itself.

For example, resolution changes with iteration number. Several different studies have recently evaluated the resolution of the NM530c system and all arrived at different values. The FWHM of sources in air at the center of the FOV are measured at $1 \mathrm{~cm}$ with 40 iteration, ${ }^{4} 7 \mathrm{~mm}$ with 60 iterations ${ }^{1}$ or $3 \mathrm{~mm}$ with 250 iterations. ${ }^{9}$ These measurements were all made at the center of the FOV, in air, but resolution also depends on where in the FOV you measure it with this camera ${ }^{5}$ and the distribution of the myocardial and background activity. ${ }^{2}$ The NM530c also inherently uses collimator modeling as part of the reconstruction algorithm and recovers resolution losses caused by the collimator geometry. Parallel-hole systems also often make use of resolution recovery (RR). ${ }^{2,10}$ Spatial resolution depends on the reconstruction used, and with RR, low-resolution parallel-hole collimators can give the same resolution as high-resolution collimators, ${ }^{11,12}$ though it may take longer (more iterations) to obtain that resolution. Finally, caution should be taken in interpreting measurements of resolution that are close to the sampling size. The CZT detector has a pixel spacing of $2.26 \mathrm{~mm}$, but when the projection data are collapsed to $128 \times 128$, the pixel size is $4.4 \mathrm{~mm}(8.8 \mathrm{~mm}$ at 64$)$. The reconstructed voxel size of the NM530c is $4 \mathrm{~mm}$. To accurately measure the FWHM of an object requires 2 to 3 samples per FWHM. Once the expected resolution of the object becomes $\leq 1 \mathrm{~cm}$, accurately measuring the resolution with voxels of $\geq 4 \mathrm{~mm}$ becomes difficult.

To further confuse the measurements, RR also alters the relationship between sensitivity and image quality. It has been shown with parallel-hole systems that RR changes the effective sensitivity of the camera, allowing similar image quality (diagnostic performance) from scans with half of the counts, acquired with the same camera and collimator. ${ }^{10}$ Increased sensitivity with general-purpose and high-sensitivity collimators leads to poor contrast to noise when compared to highresolution (HR) collimators without RR, but the same data reconstructed with RR have better contrast to noise compared to HR with (or without) RR. ${ }^{11,12}$

\section{COMPARISON OF MODERN CARDIAC IMAGING SYSTEMS}

With so many options: number of iterations, whether or not RR is used, and the effect of the activity distribution, one needs to decide how these variables should be set. A reasonable approach, then is to set the parameters at what will be used clinically. The article in this issue ${ }^{4}$ also provides data along this line with an analysis of cardiac phantoms and clinical images. In clinical images, quality can be judged with respect to clinical tasks. A useful metric, then might be the uniformity of perfusion in a normal myocardium and differences in perfusion between one system and the next. However, in evaluating a quantitative metric like this, it should be judged in a clinical context. How much non-uniformity is clinically acceptable, what difference will have a clinically significant impact? Morelle and colleagues measure a confidence interval of $\pm 15 \%$ for the differences in relative perfusion. This is for a cardiac phantom in air. But attenuation effects and background activity will influence the accuracy of the reconstruction and attenuation is known to influence the novel cardiac cameras in a different manner than does a parallel-hole system. The impact of activity from extracardiac source which are potentially outside the FOV will also alter convergence of the reconstruction and may influence noise. Thus, a $\pm 15 \%$ CI represents the 'best-case' scenario where these effects are minimized. Deciding on the clinical significance of this difference, however, would best be done with a clinical study to assess the impact on diagnostic performance.

Where does this leave us then in comparing modern cardiac imaging systems? We can try to measure clinical performance, but this is difficult and very time consuming. This approach also will be specific to a particular task and choice of acquisition and reconstruction parameters. Image quality is strongly dependent on the task to which we wish to apply the images. Quantitation is strongly influenced by partial volume effects and so might emphasize resolution, whereas lesion detection may depend on background noise character. Thus, acquisition and reconstruction should be tuned to the task at hand and different parameters used for cardiac versus, for example, oncologic imaging. However, if the camera is to be tuned for different tasks, then the metrics used to measure performance should likewise be tuned.

In the end, it is essential to consider the task for which the camera will be used and to evaluate the system performance at that task. If we want to use the camera for imaging apples, we should not evaluate its performance with oranges. The acquisition and processing of the data should be optimized for apples and then apples should be measured. Where factors are known to influence performance, these factors should be present in the study. If iterative reconstruction is to be used and is known to be influenced by background activity, then background activity should be present. Collimator modelling (resolution recovery) is known to impact on image quality and should be present during the evaluation if it will be used for the clinical task. The number of iterations used should be optimized for each system for the task under evaluation, or alternatively fixed based on a separate overriding factor such as maximum clinically 
acceptable reconstruction time. With the large diversity now available in SPECT camera design and reconstruction options, perfectly aligned comparisons may not be possible. Nevertheless, it is important to keep the comparison as equivalent and as relevant as possible. It may be that new standards are needed so that when we compare cameras for cardiac imaging, we are comparing Red Delicious to Macintosh apples and not to clementines (Figure 1).

\section{Disclosure}

R.G. Wells and T.D. Ruddy have received research grant support from GE Healthcare and Advanced Accelerator Applications. R.G. Wells has received honoraria for speaking at GE Healthcare meetings.

\section{References}

1. Imbert L, Poussier S, Franken PR, et al. Compared performance of high-sensitivity cameras dedicated to myocardial perfusion SPECT: A comprehensive analysis of phantom and human images. J Nucl Med 2012;53:1897-903. https://doi.org/10.2967/jnumed.1 12.107417.

2. Erlandsson K, Kacperski K, van Gramberg D, Hutton BF. Performance evaluation of D-SPECT: A novel SPECT system for nuclear cardiology. Phys Med Biol 2009;54:2635-49. https://doi. org/10.1088/0031-9155/54/9/003.

3. Bocher M, Blevis IM, Tsukerman L, et al. A fast cardiac gamma camera with dynamic SPECT capabilities: Design, system validation and future potential. Eur J Nucl Med Mol Imaging 2010;37:1887-902. https://doi.org/10.1007/s00259-010-1488-z.
4. Morelle M, Bellevre D, Hossein-Foucher C, et al. First comparison of performances between the new whole body cadmium-zinc-telluride SPECT-CT camera and a dedicated cardiac CZT camera for myocardial perfusion imaging. Analysis of Phantom and patients. J Nucl Cardiol 2019. https://doi.org/10.1007/s12350-019-01702-2.

5. Kennedy JA, Israel O, Frenkel A. 3D iteratively reconstructed spatial resolution map and sensitivity characterization of a dedicated cardiac SPECT camera. J Nucl Cardiol 2014;21:443-52. h ttps://doi.org/10.1007/s12350-013-9851-7.

6. Cuddy-Walsh SG, Wells RG. Patient-specific estimation of spatially variant image noise for a pinhole cardiac SPECT camera. Med Phys 2018;45:2033-47. https://doi.org/10.1002/mp.12883.

7. Timmins R, Ruddy TD, Wells RG. Patient position alters attenuation effects in multipinhole cardiac SPECT. Med Phys 2015;42:1233. https://doi.org/10.1118/1.4908015.

8. Hindorf $\mathrm{C}$, Oddstig $\mathrm{J}$, Hedeer $\mathrm{F}$, et al. Importance of correct patient positioning in myocardial perfusion SPECT when using a CZT camera. J Nucl Cardiol 2014;21:695-702. https://doi.org/10. 1007/s12350-014-9897-1.

9. Takahashi Y, Miyagawa M, Nishiyama Y, et al. Performance of a semiconductor SPECT system: Comparison with a conventional Anger-type SPECT instrument. Ann Nucl Med 2013;27:11-6. h ttps://doi.org/10.1007/s12149-012-0653-9.

10. Ali I, Ruddy TD, Almgrahi A, et al. Half-time SPECT myocardial perfusion imaging with attenuation correction. J Nucl Med 2009;50:554-62. https://doi.org/10.2967/jnumed.108.058362.

11. Lau YH, Hutton BF, Beekman FJ. Choice of collimator for cardiac SPET when resolution compensation is included in iterative reconstruction. Eur J Nucl Med. 2001;28:39-47 https://doi.org/10. 1007/s002590000387.

12. Zhang B, Zeng GL. High-resolution versus high-sensitivity SPECT imaging with geometric blurring compensation for various parallel-hole collimation geometries. IEEE Trans Inf Technol Biomed 2010;14:1121-7.

Publisher's Note Springer Nature remains neutral with regard to jurisdictional claims in published maps and institutional affiliations.' 